\title{
Equation of State of Dense Matter and Consequences for Neutron Stars
}

\author{
A. W. Thomas ${ }^{1}$, D. L. Whittenbury ${ }^{1}$, J. D. Carroll ${ }^{1}$, K. Tsushima ${ }^{1}$, and J. R. Stone ${ }^{2}$ \\ ${ }^{1}$ CSSM and ARC Centre of Excellence for Particle Physics at the Terascale, \\ School of Chemistry and Physics, University of Adelaide, Adelaide SA 5005, Australia \\ ${ }^{2}$ Department of Physics, University of Oxford, Oxford OX13PU, United Kingdom
}

\begin{abstract}
.
There is currently tremendous interest in the role of hyperons and other exotic forms of matter in neutron stars. This is particularly so following the measurement by Demorest et al. of a star with a mass almost 2 solar masses. Given that we know of no physical mechanism to stop the occurrence of hyperons at matter in beta-equilibrium above roughly 3 times nuclear matter density, we discuss the constraints on the possible maximum mass when hyperons are included in the equation of state. The discussion includes a careful assessment of the constraints from low energy nuclear properties as well as the properties of hypernuclei. The model within which these calculations are carried out is the quark-meson coupling (QMC) model, which is derived starting at the quark level.
\end{abstract}

\section{Introduction}

The recent observations of not one but two high mass neutron stars, the millisecond pulsar, PSR J1614-2230 at $1.97 \pm 0.04 M_{\odot}[6]$ and more recently of PSR J0348+0432 at $2.03 \pm 0.04 M_{\odot}$ [5], have imposed the most stringent constraints to date on the equation of state $(\mathrm{EoS})$ of dense matter. These observations have spurred re-examinations of exotic forms of matter and, in particular, led to questions of whether or not hyperons can play a significant role in interior of neutron stars, given the anticipated softening of EoS. There is no known physical mechanism preventing the appearance of hyperons in dense $\beta$-equilibriated matter and as such they should not be ignored.

The NN interaction can be fit to NN scattering data, where typically $20-30$ parameters are fit, but to fit nuclear data we also need the 3 body force, which requires additional parameters to be fit to the energy levels of light nuclei. When we want to include hyperons using this approach we run into trouble due to lack of data. There is very limited to no data on $Y N$ and $Y Y$ scattering and certainly not enough to fit the large number of parameters of a realistic potential, which suggests a different approach should be taken.

There has been a systematic study of $\Lambda$ hypernuclei and a search for $\Sigma$ hypernuclei, where contrary to reports in the 80 s there are no $\Sigma$ hypernuclei, with the exception of one very light case. The absence of medium to heavy $\Sigma$ hypernuclei means phenomenologically that the $\Sigma$ - $A$ interaction is repulsive. Its repulsive nature implies that their concentration in dense nuclear matter should be significantly reduced. This is not the case for $\Lambda$, whereas for $\Xi$ hypernuclei currently nothing is known, but experimental studies are underway at JPARC. The mass differences
$\left(M_{\Lambda}-M_{N}\right) \sim 170 \mathrm{MeV}$ and $\left(M_{\Xi}-M_{N}\right) \sim 380 \mathrm{MeV}$, suggest that for matter in $\beta$-equilibrium we could have $\Xi^{-}$and $\Lambda$ competing for dominance depending on their internal structure and its modification through interaction with the surrounding medium. This is possible since $\mu_{\Xi^{-}}=\mu_{n}+\mu_{e}$, where $\mu_{e} \sim 200 \mathrm{MeV}^{1}$.

There are many EoS available in the literature and to describe the matter in neutron stars we need an EoS which is both relativistic and includes hyperons. To separate the more realistic EoS from the rest a global approach would be to try them all and filter them by general properties extracted from terrestrial experiments and astronomical observations. While reconciling the observations with realistic model predictions is a challenging and ongoing problem we present a discussion on the constraints on the possible maximum mass when hyperons are included in the equation of state [3, 4]. Our discussion includes assessment of low energy nuclear and hypernuclear properties within a relativistic quark level model, the Quark-Meson Coupling (QMC) model. This model was used in the earlier work of Stone et al. [7], who already predicted the existence of neutron stars containing hyperons with masses as large as $2 M_{\odot}$ in 2007 . It has the advantage of being derived from the quark level, with a very small number of adjustable parameters, while being consistent with a broad range of constraints derived from hypernuclei as well as normal nuclear properties. We find that the stability under variation of the very small number of adjustable parameters is such that if a star were discovered with a mass significantly above $2.1 M_{\odot}$, we would need to consider more exotic physics, because it could not be accommodated within the current version of QMC model.

${ }^{1}$ Neutrinos neglected. 
Table 1. Meson-nucleon coupling constants determined for our baseline scenario (for which $\Lambda=0.9 \mathrm{GeV}$, and $R_{N}^{\text {free }}=1.0 \mathrm{fm}$ ) and subsequent scenarios in which differences from our standard parameter set are given in column 1 . Also shown are the saturation incompressibility, $K_{0}$; stellar radius and maximum stellar mass.

\begin{tabular}{lcccccc}
\hline Model & $g_{\sigma N}$ & $g_{\omega N}$ & $g_{\rho}$ & $\begin{array}{c}K_{0} \\
(\mathrm{MeV})\end{array}$ & $\begin{array}{c}R \\
(\mathrm{~km})\end{array}$ & $\begin{array}{c}M_{\max } \\
\left(M_{\odot}\right)\end{array}$ \\
\hline Standard & 10.42 & 11.02 & 4.55 & 298 & 12.27 & 1.93 \\
$\Lambda=1.0$ & 10.74 & 11.66 & 4.68 & 305 & 12.45 & 2.00 \\
$\Lambda=1.1$ & 11.10 & 12.33 & 4.84 & 312 & 12.64 & 2.07 \\
$\Lambda=1.2$ & 11.49 & 13.06 & 5.03 & 319 & 12.83 & 2.14 \\
$\Lambda=1.3$ & 11.93 & 13.85 & 5.24 & 329 & 13.02 & 2.23 \\
$R=0.8$ & 11.20 & 12.01 & 4.52 & 300 & 12.41 & 1.98 \\
Fock $\delta \bar{\sigma}$ & 10.91 & 11.58 & 4.52 & 285 & 12.29 & 1.98 \\
Dirac Only & 10.12 & 9.25 & 7.83 & 294 & 12.56 & 1.79 \\
Hartree Only & 10.25 & 7.95 & 8.40 & 283 & 11.85 & 1.54 \\
Nucleon Only & 10.42 & 11.02 & 4.55 & 298 & 11.64 & 2.26 \\
\hline
\end{tabular}

\section{The QMC Model}

\subsection{Background on the QMC Model}

We recall that QMC is based upon the self-consistent modification of the structure of a baryon embedded in nuclear matter. At Hartree level it involves only three adjustable parameters which describe the effective couplings of the $\sigma, \omega$ and $\rho$ mesons to the $u$ and $d$ quarks. These are fixed by adjusting them to fit the properties of symmetric nuclear matter, namely its saturation density and binding energy as well as its symmetry energy. We note that the $\sigma$ meson used here simply serves as a convenient representation of the scalar-isoscalar attraction arising from two-pion exchange.

In the most recent development of the QMC model [11], the self-consistent inclusion of the gluonic hyperfine interaction led to a very successful description of the binding energies of $\Lambda$-hypernuclei-as well as the observed absence of medium and heavy mass $\Sigma$-hypernuclei - with no additional parameters. We stress that this is achieved without any coupling of the strange quark to the $\sigma, \omega$ and $\rho$ mesons (which would be OZI suppressed) and without the need to introduce any further mesons. While the model could be supplemented with much heavier mesons containing strange quarks [12], Occam's razor suggests that one should not introduce them if they are not needed.

A clear connection has been established between the self-consistent treatment of in-medium hadron structure and the existence of many-body [13] or density dependent [15] effective forces. Dutra et al. [16] critically examined a variety of phenomenological Skyrme models of the effective density dependent nuclear force against the most up-to-date empirical constraints. Amongst the few percent of the Skyrme forces studied which satisfied all of these constraints, the Skyrme model SQMC700, derived from the QMC model, was unique in that it incorporated the effects of the internal structure of the nucleon and its modification in-medium.
While the earlier study of Stone et al. [7] demonstrated the importance of exchange (Fock) terms in calculations of the EoS of dense baryonic matter in $\beta$-equilibrium, it included only the Dirac vector term in the vector-mesonnucleon vertices. In this work we include the full vertex structure which one might expect to enhance the pressure at high density. This is especially so in the case of the $\rho$ meson for which the tensor coupling is much larger than that of the $\omega$. Our calculation[3] extends the work of Krein et al. [17], who considered nucleons only, by evaluating the full exchange terms for all octet baryons and adding them in the same way as Stone et al. [7]; as additional contributions to the energy density. Our investigation complements the important work of Miyatsu et al. who performed a relativistic Hartree-Fock calculation incorporating the tensor interaction [21]. Furthermore, we carefully explore the limit on the maximum mass of a neutron star containing hyperons while ensuring consistency with critical nuclear properties, such as the incompressibility of nuclear matter.

\subsection{Formulation of the QMC Model with Pauli Term}

As mentioned above in the QMC model one starts with a model of hadronic structure such as the MIT bag $[8,9]$ (or NJL [10]) and solves self consistently for the internal structure of the baryons in medium. This can then be parametrized [11] by a polynomial in $g_{\sigma N}(0) \bar{\sigma}$,

$$
M_{B}^{*}=M_{B}-w_{\sigma B} g_{\sigma} \bar{\sigma}+\tilde{w}_{\sigma B} \frac{d}{2}\left(g_{\sigma} \bar{\sigma}\right)^{2}
$$

where the weightings $w_{\sigma B}$ and $\tilde{w}_{\sigma B}$ simply allow the use of a unique coupling to nucleons, $g_{\sigma N}(0)=g_{\sigma N}=g_{\sigma}$. The response of the baryons internal structure to the scalar field is of great interest. This modification in medium develops a natural non-linear dependence (in this version, through the bag model equations) on the scalar field giving rise to the scalar polarisability, $d$, which can be interpreted as the physical origin of the non-linear scalar potential in non-linear versions of Quantum Hadro-dynamics 
(QHD)[2]. The consequence of polarisability in atomic physics is many body forces and the same is true in nuclear physics [13, 14].

The baryon energy density, $\epsilon_{B}$, in the QMC model is then given by

$$
\epsilon_{B}=\frac{2}{(2 \pi)^{3}} \sum_{B} \int_{|\mathbf{p}|<p_{F}} d \mathbf{p} \sqrt{p^{2}+M_{B}^{* 2}}
$$

where the effective, in-medium baryon masses, $M_{B}^{*}$, are calculated self-consistently for an MIT bag immersed in (and in Ref. [11], parameterized as functions of) a mean scalar field, designated here by a barred symbol. At a given density, $\bar{\sigma}$ is self-consistently expressed as

$$
\bar{\sigma}=-\frac{2}{m_{\sigma}^{2}(2 \pi)^{3}} \sum_{B} \int_{|\mathbf{p}|<p_{F}} d \mathbf{p} \frac{M_{B}^{*}}{\sqrt{p^{2}+M_{B}^{* 2}}} \frac{\partial M_{B}^{*}}{\partial \bar{\sigma}} .
$$

An additional contribution, $\delta \bar{\sigma}$, to the scalar field arises if we include the Fock terms in the minimization of the energy density. This provides only a small correction to the mean field, and its effect is included only in the scenario denoted $\delta \bar{\sigma}$. The total hadronic energy density, $\epsilon_{H}$, is the sum of baryonic, $\epsilon_{B}$, and mesonic, $\epsilon_{\sigma \omega \rho \pi}$ contributions, for which

$$
\begin{aligned}
\epsilon_{\sigma \omega \rho \pi}= & \sum_{\alpha=\sigma, \omega, \rho} \frac{1}{2} m_{\alpha}^{2} \bar{\alpha}^{2} \\
& +\sum_{\alpha=\sigma, \omega, \rho, \pi} \sum_{B B^{\prime}} \frac{C_{B B^{\prime}}^{\alpha}}{(2 \pi)^{6}} \iint_{\substack{|\mathbf{p}|<p_{F} \\
\left|\mathbf{p}^{\prime}\right|<p_{F^{\prime}}}} d \mathbf{p} d \mathbf{p}^{\prime} \boldsymbol{\Xi}_{B B^{\prime}}^{\alpha}(4)
\end{aligned}
$$

where $C_{B B^{\prime}}^{\sigma}=C_{B B^{\prime}}^{\omega}=\delta_{B B^{\prime}} \cdot C_{B B^{\prime}}^{\rho}$ and $C_{B B^{\prime}}^{\pi}$, which arise from symmetry considerations, are given in Ref. [7]. Note that the $\pi$ meson only contributes to the second term in Eq. (4) as it is coupled via a pseudo-vector current. For $\epsilon_{\sigma \omega \rho}$, the integrand has the form

$$
\boldsymbol{\Xi}_{B B^{\prime}}^{m}=\frac{1}{2} \sum_{s, s^{\prime}}\left|\bar{u}_{B^{\prime}}\left(p^{\prime}, s^{\prime}\right) \Gamma_{m B} u_{B}(p, s)\right|^{2} \Delta_{m}(\mathbf{k}),
$$

where $\Delta_{m}(\mathbf{k})$ is the Yukawa propagator for meson $m$ with momentum $\mathbf{k}=\mathbf{p}-\mathbf{p}^{\prime}$. For the vector mesons, the full vertex structure is included in the manner of Ref. [17] as

$$
\begin{aligned}
\Gamma_{\sigma B} & =g_{\sigma B} C_{B}(\bar{\sigma}) F^{\sigma}\left(k^{2}\right) \mathbf{1}=-\frac{\partial M_{B}^{*}}{\partial \bar{\sigma}} F^{\sigma}\left(k^{2}\right) \mathbf{1},(6) \\
\vec{\Gamma}_{\eta B} & =\epsilon_{\eta}^{\mu} \vec{\Gamma}_{\mu \eta B} \\
& =\epsilon_{\eta}^{\mu}\left[g_{\eta B} \gamma_{\mu} F_{1}^{\eta}\left(k^{2}\right)+\frac{i f_{\eta B} \sigma_{\mu v}}{2 M_{B}^{*}} k^{\nu} F_{2}^{\eta}\left(k^{2}\right)\right] \vec{\tau}(8) \\
\vec{\Gamma}_{\pi B} & =i \frac{g_{A}}{2 f_{\pi}} F^{\pi}\left(k^{2}\right) \gamma^{\mu} k_{\mu} \gamma_{5} \vec{\tau},
\end{aligned}
$$

with the isospin matrix only applicable to isovector mesons and $\eta \in\{\omega, \rho\}$.

As usual, the effect of short distance repulsion on the Fock terms is simulated by the replacement

$$
\frac{\vec{q}^{2}}{\left(\vec{q}^{2}+m^{2}\right)} \rightarrow 1-\frac{m^{2}}{\left(\vec{q}^{2}+m^{2}\right)}
$$

from which the unit term is subtracted, thus eliminating a $\delta$-function. The form factors $F_{1,2}^{\alpha}$ all have the same dipole form with the cutoff mass $\Lambda$ varied from 0.9 to $1.3 \mathrm{GeV}$ to test the sensitivity. In our standard scenario we take $\Lambda=0.9 \mathrm{GeV}$ and the ratio of tensor to vector couplings $\left(f_{\rho N} / g_{\rho N}=3.70\right)$ consistent with values derived within QMC. These ratios correspond to those from vector meson dominance.

Of the baryon-meson coupling constants $g_{\sigma B}(\bar{\sigma}), g_{\omega B}$, and $g_{\rho B}$, only $g_{\sigma B}$ is density dependent. Its model parameterisation [11] is dependent on the free nucleon radius, which is taken to be $R_{N}^{\text {free }}=1.0 \mathrm{fm}$ - with an alternate scenario having $R_{N}^{\text {free }}=0.8 \mathrm{fm}$. The density dependence is given by

$$
\frac{\partial}{\partial \bar{\sigma}}\left[g_{\sigma B}(\bar{\sigma}) \bar{\sigma}\right]=g_{\sigma B} C_{B}(\bar{\sigma})=-\frac{\partial M_{B}^{*}}{\partial \bar{\sigma}} .
$$

Values of the coupling constants $g_{\alpha N}$ for various mesons $\alpha$ and a selection of scenarios considered in this work are presented in Table 1 . The couplings $g_{\omega B}$ and $g_{\rho B}$ are expressed in terms of the quark level couplings

$$
g_{\omega B}=n_{u, d}^{B} g_{\omega}^{q} ; g_{\rho B}=g_{\rho N}=g_{\rho}^{q},
$$

where $n_{u, d}^{B}$ represents the number of light quarks in baryon $B$. The $\sigma, \omega$ and $\rho$ couplings to the quarks are constrained to reproduce a saturation energy per baryon of $\mathcal{E}_{\text {sat }}=-15.86 \mathrm{MeV}$ and an asymmetry energy coefficient of $a_{\text {asym }}=32.5 \mathrm{MeV}$ at the saturation density $n_{0}=$ $0.16 \mathrm{fm}^{-3}$. The $\omega, \rho$ and $\pi$ masses are constrained to their experimental values, whereas the $\sigma$ mass is taken to be $700 \mathrm{MeV}$.

For a compact object in $\beta$-equilibrium we solve the familiar system of equations for the number densities of the baryons and leptons [19]. The lepton energy density and pressure are given by the usual formulas for a degenerate Fermi gas. In order to obtain the neutron star properties shown in Table 1, we solve the Tolman-Oppenheinmer-Volkov equations for the gravitational mass and radius [19]. The resulting dependence of the neutron star mass on radius, for a selection of the variations of the model, is shown in Fig. 2.

\section{Discussion}

In Fig. 1 we present the pressure versus total baryon density as calculated within the QMC model for both symmetric (Top) and pure neutron (Bottom) matter. The shaded regions have been inferred from a comparison of experimental data on matter flow in energetic heavy ion collisions by Danielewicz et al. (see Ref. [1] and references therein). In both cases the QMC model predicts somewhat higher pressure at a given density than the extrapolation of the transport theory. This may not be surprising because the QMC model and the model used in Ref. [1] are based on very different physics and make very different assumptions.

In Table 1 we present the coupling constants, incompressibilities of symmetric nuclear matter, and stellar 


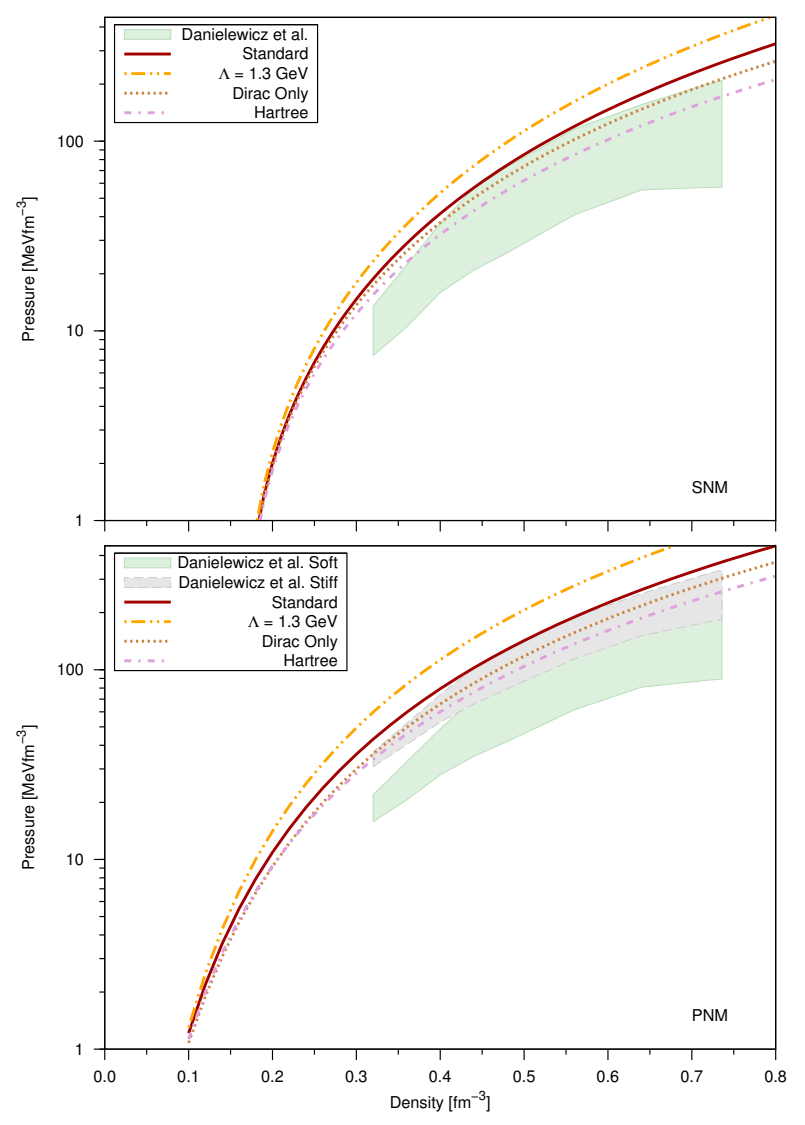

Figure 1. (Color online) (Top) Pressure in SNM as a function of density as predicted in the QMC model. The shaded area is taken from [1]. (Bottom) The same as (Top) but for PNM. The upper and lower shaded areas correspond to two different estimates of the contribution of the symmetry pressure to the total pressure. For more detail see [1].

properties, for a number of variations of the QMC model, in each scenario including the $\sigma, \pi, \omega$ and $\rho$ Fock terms. We note that in all scenarios $\Xi^{-}$hyperons are present in significant quantities in the the maximum mass stars.

It is remarkable that in all of the scenarios investigated, the stellar properties are largely consistent, and similar to those reported by Stone et al. [7]. Scenarios in which the maximum stellar mass lies outside of the range 1.9$2.14 M_{\odot}$ correspond to nuclear matter compressibilities above the upper limit set in the recent comprehensive analysis of giant monopole resonance data [20]. While this cannot be true in general, it is certainly the case for the QMC model.

Turning to the effects of the inclusion of the full exchange terms on stellar properties, we find that the threshold density for $\Xi^{-}$is lowered, while those of $\Lambda$ and $\Xi^{0}$ are raised, as demonstrated in Fig. 3. In all scenarios there is a greater splitting between the thresholds of the $\Xi$ baryons than that found by Stone et al. [7].

In our standard or baseline scenario, the $\Xi^{-}$threshold occurs at $0.42 \mathrm{fm}^{-3}$, followed by $\Xi^{0}$ at $0.91 \mathrm{fm}^{-3}$. We find that $\Lambda$ production is not energetically favoured at densities

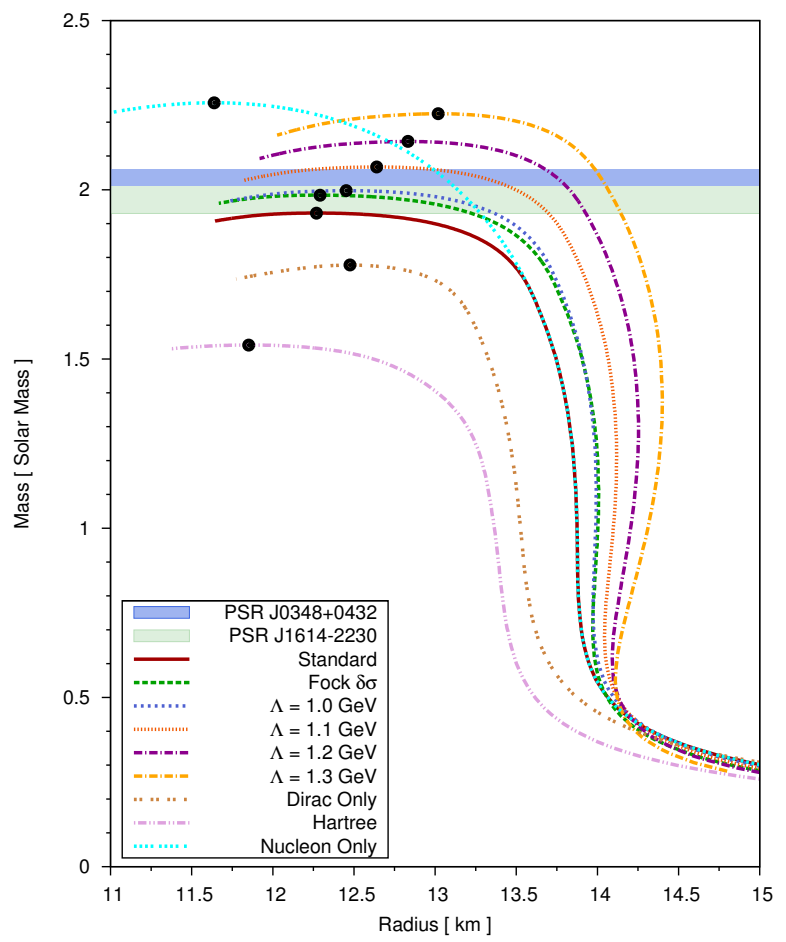

Figure 2. Gravitational Mass versus radius relationship for various scenarios described in the text. The black dots represent maximum mass stars and the coloured bars represent observed pulsar constraints. At low density the BPS [24] EoS is used.

considered here, in agreement with Ref. [21]. Similarly, it is insensitive to the choice of free nucleon radius, despite a moderate impact on the couplings.

The correction $(\delta \bar{\sigma})$ to the scalar mean field arising from the Fock terms decreases the incompressibility by $13 \mathrm{MeV}$, yet other observables remain largely unaltered by this addition. The cutoff, $\Lambda$, used in form factors (which controls the strength of the Fock terms) exhibits a more pronounced relationship with the observables in Table 1. Increasing $\Lambda$ beyond $0.9 \mathrm{GeV}$ raises the incompressibility with the case denoted $\Lambda=1.3 \mathrm{GeV}$ already exceeding the limit of $K<315 \mathrm{MeV}$. We stress that $\Lambda$ could not take a lower value without impacting the masses of the $\sigma, \omega$, and $\rho$. The $\pi$, however, has a much lower mass and as such could involve a lower cutoff. We investigated this possibility but found only a minimal effect on the EoS, as expected from the small contribution the pion makes to the EoS at high density[3]. Overall, increases in the cutoff correlate with increases in both the saturation incompressibility and maximum stellar mass.

We stress that the QMC model does not predict the appearance of $\Sigma$ hyperons at any density where the model can be considered realistic. This is in contrast to a number of other relativistic models which do predict the $\Sigma$ threshold to occur, even prior to that of the $\Lambda[22,23]$. We note that Schaffner-Bielich [22] considered a phenomenological modification of the $\Sigma$ potential with additional repulsion, which significantly raised its threshold density. In the case of the QMC model the physical explanation of the ab- 


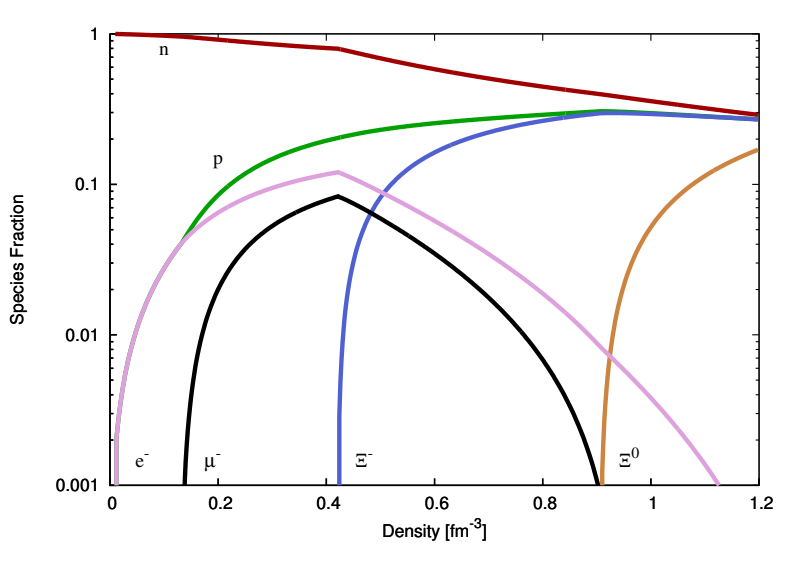

Figure 3. (Color online) Species fraction as a function of baryon number density in GBEM, using the standard scenario EoS.

sence of $\Sigma$-hyperons is very natural, with the mean scalar field enhancing the repulsive hyperfine force in the bound $\Sigma$. Recall that the hyperfine splitting is due to one-gluonexchange, which determines the free $\Sigma-\Lambda$ mass splitting in the MIT bag model.

It is worth remarking that upon inclusion of the tensor coupling, the proton fraction increases more rapidly as a function of total baryon density. Thus the maximum electron chemical potential is increased, making the $\Xi^{-}$more energetically favourable. This is likely to increase the probability of the direct URCA cooling process in protoneutron stars. As a further consequence, it may well influence the production of $\pi^{-}$and $\bar{K}$ condensates. Changes to the $\Lambda$ threshold (occurs at higher density with lower maximum species fraction) reduces the possibility of $\mathrm{H}$ dibaryon production as constrained by $\beta$-equilibrium of chemical potentials.

For comparison purposes, we also include a 'Nucleon Only' scenario, in which hyperons are artificially excluded. In this case the EoS is increasingly stiffer at densities above $0.4 \mathrm{fm}^{-3}$, leading to a large maximum stellar mass of $2.26 M_{\odot}$, consistent with many other nucleon-only models.

\section{Summary}

In summary, taking into account the full tensor structure of the vector-meson-baryon couplings in a Hartree-Fock treatment of the QMC model gives increased pressure at high density - largely because of the $\rho N$ tensor coupling - while maintaining reasonable values of incompressibility at saturation density. The conceptual separation between the incompressibility at saturation density and the amount of pressure or 'stiffness' at higher densities is critical. It is the latter that leads to neutron stars with maximum masses ranging from $1.90 M_{\odot}$ to $2.14 M_{\odot}$, even when allowance is made for the appearance of hyperons. This suggests that hyperons are very likely to play a vital role as consituents of neutron stars.

\section{Acknowledgements}

This work was supported by the University of Adelaide and the Australian Research Council through an Australian Laureate Fellowship (AWT, FL0992247) and through the ARC Centre of Excellence for Particle Physics at the Terascale. KT was supported in part by a visiting professorship at IIP (Brazil).

\section{References}

[1] P. Danielewicz, R. Lacey and W. G. Lynch, Science 298, 1592 (2002)

[2] K. Saito, K. Tsushima and A. W. Thomas, Prog. Part. Nucl. Phys. 58, 1 (2007) [hep-ph/0506314].

[3] D. L. Whittenbury et al,arXiv:1204.2614 [nucl-th].

[4] I. Bednarek, P. Haensel, J. L. Zdunik, M. Bejger and R. Manka, Astronomy and Astrophysics, 543, A157 (2013)

[5] J. Antoniadis et al., Science 340, 6131 (2013)

[6] P. Demorest et al., Nature 467 (2010) 1081.

[7] J. Rikovska Stone et al., Nucl. Phys. A 792 (2007) 341

[8] P. A. M. Guichon, Phys. Lett. B 200, 235 (1988).

[9] P. A. M. Guichon et al., Nucl. Phys. A 601, 349 (1996)

[10] W. Bentz and A. W. Thomas, Nucl. Phys. A 696, 138 (2001) [nucl-th/0105022].

[11] P. A. M. Guichon, A. W. Thomas and K. Tsushima, Nucl. Phys. A 814, 66 (2008).

[12] S. Weissenborn, D. Chatterjee and J. SchaffnerBielich, Phys. Rev. C 85, 065802 (2012)

[13] P. A. M. Guichon and A. W. Thomas, Phys. Rev. Lett. 93, 132502 (2004)

[14] A. W. Thomas, Nucl. Phys. A 787, 396 (2007).

[15] P. A. M. Guichon et al., Nucl. Phys. A 772, 1 (2006)

[16] M. Dutra et al., Phys. Rev. C 85, 035201 (2012).

[17] G. Krein, A. W. Thomas and K. Tsushima, Nucl. Phys. A 650, 313 (1999)

[18] T. A. Rijken, M. M. Nagels and Y. Yamamoto, Prog. Theor. Phys. Suppl. 185, 14 (2010).

[19] N. K. Glendenning, "Compact stars: Nuclear physics, particle physics, and general relativity," New York, USA: Springer (1997)

[20] J. R. Stone, S. A. Moszkowski and N. J. Stone, to be published.

[21] T. Miyatsu, T. Katayama and K. Saito, Phys. Lett. B 709, 242 (2012)

[22] J. Schaffner-Bielich, Nucl. Phys. A 835, 279 (2010)

[23] F. Weber, Prog. Part. Nucl. Phys. 54, 193 (2005)

[24] G. Baym, C. Pethick and P. Sutherland, Astrophys. J. 170, 299 (1971). 